\title{
New MeSH for Sedentary Behavior
}

\section{Brigid M. Lynch, Charles E. Matthews, and Katrien Wijndaele on behalf of the Sedentary Behaviour Council of the International Society for Physical Activity and Health}

To the editor,

MeSH (Medical Subject Headings) is the (United States) National Library of Medicine's comprehensive, controlled vocabulary developed for the purpose of indexing journal articles for MEDLINE. It is a set of terms akin to a thesaurus, arranged in a hierarchical structure that enables MEDLINE searches at various levels of specificity. Systematic reviews that utilize MEDLINE rely heavily on the database's controlled vocabulary to identify relevant publications.

One of the first projects initiated by the Sedentary Behaviour Council of the International Society for Physical Activity and Health, after the Council's formation in November 2016, was a submission to the National Library of Medicine to add MeSH terms that describe sedentary behavior. Prior to the Council's submission, there had been no clear vocabulary useful for indexing and retrieving sedentary behavior-related research from the biomedical literature.

After reviewing the terminology used across numerous systematic reviews of sedentary behavior research and consultation with members of the Sedentary Behaviour Council's Reference Group, 3 new MeSH terms were proposed, as well as a revision to an existing MeSH term. The submission was made via the National Library of Medicine's Web page for new suggestions (https://www. nlm.nih.gov/mesh/meshsugg.html) in November 2017.

The new MeSH terms proposed were as follows: Sedentary behavior (which we defined as "any waking behavior characterized by an energy expenditure $\leq 1.5$ metabolic equivalents (METs), while in a sitting, reclining or lying posture"), Sitting (defined as "a position in which one's weight is supported by one's buttocks rather than one's feet, and in which one's back is upright"), and Screen time (defined as "the time spent on screen-based behaviors").

The Sedentary Behaviour Council's submission also suggested that the existing MeSH term Sedentary lifestyle to be changed to Physical inactivity. Sedentary lifestyle was defined by the National Library of Medicine as: "Usual level of physical activity that is less than 30 minutes of moderate-intensity activity on most days of the week." We also suggested that this definition was changed to: "Not achieving 150 minutes of moderate-tovigorous-intensity physical activity per week or 75 minutes of vigorous-intensity physical activity per week or an equivalent combination of moderate- and vigorous-intensity activity."

\section{Additions to MeSH From January 2019}

The National Library of Medicine reviews suggested changes and releases an updated MeSH vocabulary in November each year, for implementation from the following January. In November 2018, the additional MeSH terms were added: Sedentary behavior (defined as "behaviors during waking hours that have low energy expenditure and are often performed in a sitting or reclining posture"), Sitting position (no scope note was provided, but the broad concept is defined as "putting the weight of the body against the ground or a horizontal object such as a chair seat"), and Screen time ("period of activities done in front of an electronic screen, such as watching television, working on a computer, or playing video games"). These new MeSH terms will not be retrospectively applied but will be added to new research published in MEDLINE from January 2019 onward.

The MeSH term Sedentary lifestyle remained unaltered.

The inclusion of these additional MeSH terms will facilitate more efficient searching within MEDLINE and improve the rigor of systematic review search strategies.

\section{Acknowledgments}

B.M.L. is supported by a Mid-Career Fellowship (MCRF18005) from the Victorian Cancer Agency.
Lynch is with the Cancer Epidemiology Division, Cancer Council Victoria, Melbourne, VIC, Australia; the Centre for Epidemiology and Biostatistics, Melbourne School of Population and Global Health, The University of Melbourne, VIC, Australia; and the Physical Activity Laboratory, Baker Heart \& Diabetes Institute, Melbourne, VIC, Australia. Matthews is with the Metabolic Epidemiology Branch, Division of Cancer Epidemiology \& Genetics, National Cancer Institute, Bethesda, MD, USA. Wijndaele is with the MRC Epidemiology Unit, Institute of Metabolic Science, School of Clinical Medicine, University of Cambridge, Cambridge, United Kingdom. Lynch (brigid.lynch@cancervic.org.au) is corresponding author. 